\title{
Can foveal avascular zone (FAZ) assessment by OCT angiography, used for grading of diabetic retinopathy?
}

\begin{abstract}
The most widely used grading system for Diabetic Retinopathy (DR) is International Clinical Disease Severity Scale for DR. A qualitative scale that is based on clinical findings and grade DR as (mild, moderate, sever) non-proliferative and proliferative. Disadvantages of this grading system can be listed as missing subtle details in exam may change the grade; could not differentiate those who are at higher risk in each grade; is not well correlated to visual function; could not help to choose more appropriate treatment modality; could not be used to follow the treatment effects. Adding quantitative values to grading system may alleviate these shortages. The OCT angiography, as a novel imaging modality, has eased qualitative and quantitative retinal assessment. Among indexes, the FAZ metrics showed most clinical relevance with progression of disease; with visual function; to guide selection of the more appropriate treatment modality and to follow its' effect up. These propose the FAZ as a potential index to be used for grading purposes. Before introduction of the OCTA, it was not feasible to assess the FAZ metrics, accurately.
\end{abstract}

Keywords: diabetic retinopathy, optical coherence tomography angiography, foveal avascular zone, fluorescein angiography
Volume 5 Issue 3 - 2016

\author{
Joobin Khadamy \\ Eye Research Center, Rassoul Akram hospital, Iran University of \\ Medical Sciences (IUMS), Iran
}

Correspondence: Joobin Khadamy, Eye Research Center, Rassoul Akram hospital, Sattarkhan-Niayesh Street, Tehran |4456-|3| 3|, Iran, Tel +989|25394802, Fax 982 |66509|62, Email joobin_kh@hotmail.com

Received: December 04, 2016 | Published: December 06, 2016

\begin{abstract}
Abbreviations: DR, diabetic retinopathy; WHO, world health organization; DM, diabetes mellitus; FA, fluorescein angiography; OCTA, optical coherence tomography angiography; IRMA, intraretinal microvascular abnormalities; NV, neovascularization; FAZ, foveal avascular zone

\section{Opinion}

Diabetic retinopathy (DR) not only is common among diabetics, but also is leading cause of new onset blindness in developed countries. Fortunately, visual loss is preventable by early detection and treatment of DR. Current screening guidelines suggest yearly to monthly full dilated fundus exam by ophthalmologist, based on DR grade. The World Health Organization (WHO) estimated that the number of individuals with diabetes mellitus (DM) to raise to 360 million by the year 2030. ${ }^{1}$ This number of affected individuals will be a burden on eye care health system which calls for computerized and digitalized screening and grading of patients. Wider field imaging systems have shown to be comparative to clinical examinations. ${ }^{2}$ But still grading of these images is qualitative and needed to be reviewed by specialists.
\end{abstract}

Functional imaging systems such as the fluorescein angiography (FA), mainly provide 2 dimensional images of retina which grant information regarding blood circulation and integrity of blood vessels. The FA is considered as gold standard for assessing retinal vasculture but also mainly is a qualitative assessment. The FA is considered invasive, as preformed after intravenous injection of dye. Dye exposes patients to possible deadly reactions and also is contraindicated in some systemic diseases. The FA cannot fully assess deeper structures such as choroidal vasculture. By introduction of Optical Coherence Tomography Angiography (OCTA) that provides qualitative and quantitative data regarding the retina, choroid and vascular structures; There is promises that the OCTA may play a unique role in predicting, screening, grading, following up and also guiding the treatment of DR. The OCTA in contrast to FA, is not invasive. The OCTA uniquely can visualize the changes in superficial and deeper layers, separately. These information may guide the laser wavelength selection and treatment localization. Fortunately, the combined OCTA and FA/ ICG imaging modules are now available. This will add functional information to OCTA images. There are attempts to conjunct OCT and laser tissue coagulation systems in other areas of medicine. ${ }^{3}$ Conjunction of combined OCTA and FA/ICG imaging systems with laser photocoagulation systems may be the perspective of robotic laser treatment of diabetic retinopathy!

Grading of diseases is essential to reach a consensus among clinicians to design harmonized follow up and treatment guidelines. The most commonly used grading system for DR is International Clinical Disease Severity Scale for DR. ${ }^{4}$ Grades include "no apparent retinopathy"; "mild non-proliferative retinopathy (NPDR)" which is characterized by the presence of a few microaneurysms; "moderate NPDR" which is characterized by the presence of microaneurysms, intraretinal hemorrhages or venous beading that do not reach the sever grade; "severe NPDR" which is diagnosed based on presence of the 4 quadrants of hemorrhages: 2 or more quadrants of venous beading :1 or more quadrants intraretinal microvascular abnormalities (IRMA) rule of the ETDRS; and "proliferative diabetic retinopathy (PDR)" which is characterized by neovascularization(NV). 
Knowing that $50 \%$ of those with sever NPDR are at risk of developing to PDR, ${ }^{5}$ will add to the importance of meticulous fundus exam. Missing subtle vascular abnormality such as a single IRMA or tuft of NV may change the assumption of the grade of DR, which may threaten patients' vision due to delays in follow up and treatment. Furthermore, severity could not be quantitatively expressed in each grade. Therefore, this qualitative grading cannot distinguish individuals with no apparent retinopathy who are at greater risk of developing retinopathy; some of those who may need more frequent visit than current guidelines. Moreover, this grading is not essentially correlated to patients' visual acuity. ${ }^{6}$ Patients with PDR may also have a 20/20 vision! Additionally, treatment effects such as reperfusion and reduced capillary non-perfusion could not be expressed qualitatively or quantitatively in this grading. All of these highlight disadvantages of this qualitative grading system. Adding quantitative information to qualitative grading scales may resolve these issues. The OCTA imaging systems that provide both quantitative and qualitative information, could be used for grading purposes in future.

The foveal avascular zone (FAZ) is capillary free area encircled by foveal capillaries ring. It is located in the center of macula where most perfused and visually sensitive part of retina is. Any changes in structure or perfusion of this location are reflected in vision of patient. The FAZ enlargement can be used as an index of non-perfusion which could be translated to ischemia of central retina. The FAZ was first described in vivo by FA. ${ }^{7}$ The FAZ shape is identical in the OCTA and FA, and the FAZ size was correlated in both. ${ }^{8}$ The OCTA believed to be more accurate than the FA to demarcate and measure the FAZ; as it is not obscured by leakages, pooling or staining from fluorescein. However, shadows from hemorrhage and macular edema may affect the FAZ measurements by the OCTA.

Additionally, the OCTA enables us to measure and define FAZ characteristics across the three superficial (SCP), middle (MCP) and deep (DCP) capillary plexuses which could not be assessed by means of the FA or SD-OCT. These two just depict the FAZ in superficial slab. Our center reviewed literature regarding the OCTA applications in diabetics which is going to be published soon. Available reports are few, but interesting facts regarding the FAZ is published..$^{7-18}$ The FAZ in normal individual was described in the OCTA as an intact well-demarcated round or oval area of reduced capillary density with a border without gaps, holes or interruption of the capillary network in both superficial and deep plexuses. Its' normal longest diameter is located either on vertical or horizontal axis. ${ }^{9}$ The FAZ diameters in normal individuals are similar in SCP, MCP and DCP. ${ }^{18}$ In diabetics, the FAZ is enlarged due to vasculture disintegrity. ${ }^{9}$ The FAZ often appeared to be asymmetrical due to gaps, holes or interruption of the capillary networks which form an irregular circles with hypo reflection that demarcates the enlarged FAZ. ${ }^{8,9}$

Maximum diameter of the FAZ was usually neither on the horizontal nor on the vertical axis. ${ }^{9}$ Additionally, The FAZ diameters is not the same in SCP, MCP and DCP. ${ }^{18}$ The FAZ was best distinguished at the MCP while the FAZ enlargement was more pronounced in the deep layer in the DR group. ${ }^{7,16}$ This finding may also add to our knowledge regarding pathophysiology behind the ischemic changes in DR. The OCTA has increased feasibility of qualitative FAZ assessment; but the most impressing are regarding the quantitative FAZ assessments the FAZ size increased significantly by progression of retinopathy grade in almost all reports..$^{9-17}$ (Table 1) has summarized current available range of the mean FAZ size values in different grades of disease.
Table does not isolate values in superficial and deep plexuses. For further details, one may refer to references or wait for our review article to be published.

Table I The FAZ size correlations with diabetic retinopathy grade in reviewed articles

\begin{tabular}{lll}
\hline Subjects & $\begin{array}{l}\text { Mean diameter in } \\
\text { micron (Range) }\end{array}$ & $\begin{array}{l}\text { Mean Area in } \\
\mathbf{m m}^{2} \text { (Range) }\end{array}$ \\
\hline $\begin{array}{l}\text { Healthy } \\
\text { individual }\end{array}$ & $(350-659)$ & $(0.25-0.40)$ \\
$\begin{array}{l}\text { Diabetics with } \\
\text { no DR }\end{array}$ & $(370-696)$ & $(0.34-0.54)$ \\
NPDR & $(370-813)$ & $(0.40-0.46)$ \\
PDR & $(410-1,150)$ & -0.47 \\
\hline
\end{tabular}

Furthermore, the FAZ is enlarged in diabetics not only before developing to apparent DR but even before systemic diagnosis of DM, so it could also be used as a predictor for whom may need closer follow up. ${ }^{11,15}$ Putting these findings alongside our previous knowledge regarding the FAZ characteristics such as being considered as a vascular index and correlated to presumed ischemic pathophysiology behind DR; being correlated with VA in DR;;,19 being able to help treatment selection as DME patients with larger FAZ was reported to be non-responder to laser therapy, who may benefit more from AntiVEGF treatments; ${ }^{20}$ being influenced by treatment modalities (laser or Anti-VEGF therapies) that may lead to reperfusion and reduce the FAZ area: ${ }^{17}$ these altogether propose the FAZ characteristics as a potential qualitative and quantitative index that not only can be used to grade the DR but also to predict DR, guide the selection of appropriate treatment, help to localize laser treatment targets and follow up the effect of treatment. It should be pointed out that before introduction of the OCTA, the FAZ qualitative and quantitative specifications could not be accurately and reliably assessed.

The OCTA can help us to quantitatively grade changes which presumed to be superior or complementary to current qualitative grading systems. It should be emphasized that there are massive data offered by OCTA which are not limited to the FAZ metrics but their clinical relevance are not fully investigated. While there are continuous efforts among developers to evolve the OCTA device technology to reduce its limitations, there should be effort to apply the enormous data retrieved from analyzing the images to our daily clinical practice. As current OCTA reports are small in size, so further larger scale investigations which are focused on the FAZ assessment in normal population and various grades of DR are warranted.

In summary, the FAZ characteristics make it a potentially unique index to be applied in management of DR. Aiming this, further efforts are necessitated to define cut off points for normal individuals and each grade of DR, cut off points as a threshold for considering the treatment and also cut off points that help to select the modality of choice for treatment. Besides these, assessing the FAZ changes after current treatment modalities (laser therapy or Anti-VEGF therapies) by the OCTA, are of special importance. The OCTA as a single modality or joint with the FA in combined modalities, shows promises to be considered as cornerstone of DR management to predict, grade, guide the treatment and follow it up. 


\section{Funding}

None.

\section{Acknowledgments}

None.

\section{Conflicts of interest}

The authors declare that there was no conflict of interest.

\section{References}

1. Wild S, Roglic G, Green A, et al. Global prevalence of diabetes: estimates for the year 2000 and projections for 2030. Diabetes care. 2030;27(5):1047-1053.

2. Ghasemi Falavarjani K, Wang K, Khadamy J, et al. Ultra-wide-field imaging in diabetic retinopathy; an overview. J Curr Ophthalmol. 2016;28(2):57-60.

3. Kelly KM, Svaasand LO, Nelson JS. Optimization of laser treatment safety in conjunction with cryogen spray cooling. Arch Dermatol. 2003;139(10):1372-1373.

4. Wilkinson CP, Ferris FL, Klein RE, et al. Proposed international clinical diabetic retinopathy and diabetic macular edema disease severity scales. Ophthalmology. 2003;110(9):1677-1682.

5. Ferris F. Early photocoagulation in patients with either type I or type II diabetes. Trans Am Ophthalmol Soc. 1996;94:505-537.

6. Bengtsson B, Heijl A, Agardh E. Visual fields correlate better than visual acuity to severity of diabetic retinopathy. Diabetologia. 2005;48(12):2494 2500 .

7. Laatikainen L, Larinkari J. Capillary-free area of the fovea with advancing age. Invest Ophthalmol Vis Sci. 1977;16(12):1154-1157.

8. Yu S, Lu J, Cao D, et al. The role of optical coherence tomography angiography in fundus vascular abnormalities. BMC Ophthalmol. 2016;16:107.

9. Freiberg FJ, Pfau M, Wons J, et al. Optical coherence tomography angiography of the foveal avascular zone in diabetic retinopathy. Graefes Arch Clin Exp Ophthalmol. 2016;254(6):1051-1058.
10. Choi w, waheed nk, moult em, et al. ultrahigh speed swept source optical coherence tomography angiography of retinal and choriocapillaris alterations in diabetic patients with and without retinopathy. Retina. 2016.

11. Takase N, Nozaki M, Kato A, et al. Enlargement of Foveal Avascular Zone in Diabetic Eyes Evaluated by En Face Optical Coherence Tomography Angiography. Retina. 2015;35(11):2377-2383.

12. Al-Sheikh M, Akil H, Pfau M, et al. Swept-Source OCT Angiography Imaging of the Foveal Avascular Zone and Macular Capillary Network Density in Diabetic Retinopathy. Invest Ophthalmol Vis Sci. 2016;57(8):3907-3913.

13. Cennamo G, Romano MR, Nicoletti G, et al. Optical coherence tomography angiography versus fluorescein angiography in the diagnosis of ischaemic diabetic maculopathy. Acta Ophthalmol. 2016.

14. Bhanushali D, Anegondi N, Gadde SG, et al. Linking Retinal Microvasculature Features With Severity of Diabetic Retinopathy Using Optical Coherence Tomography Angiography. Investigative ophthalmology \& visual science. 2016;57(9):519-525.

15. de Carlo TE, Chin AT, Bonini Filho MA, et al. Detection of Microvascular Changes in Eyes of Patients with Diabetes but Not Clinical Diabetic Retinopathy Using Optical Coherence Tomography Angiography. Retina. 2015;35(11):2364-2370.

16. Di G, Weihong Y, Xiao Z, et al. A morphological study of the foveal avascular zone in patients with diabetes mellitus using optical coherence tomography angiography. Graefes Arch Clin Exp Ophthalmol. 2016;254(5):873-879

17. Miwa Y, Murakami T, Suzuma K, et al. Relationship between Functional and Structural Changes in Diabetic Vessels in Optical Coherence Tomography Angiography. Sci Rep. 2016;6:29064.

18. Park JJ, Soetikno BT, Fawzi AA. Characterization of the Middle Capillary Plexus Using Optical Coherence Tomography Angiography in Healthy and Diabetic Eyes. Retina. 2016;36(11):2039-2050.

19. Balaratnasingam $\mathrm{C}$, Inoue $\mathrm{M}, \mathrm{Ahn} \mathrm{S}$, et al. Visual Acuity Is Correlated with the Area of the Foveal Avascular Zone in Diabetic Retinopathy and Retinal Vein Occlusion. Ophthalmology. 2016;123(11):2352-2367.

20. Lee J, Moon BG, Cho AR, et al. Optical Coherence Tomography Angiography of DME and Its Association with Anti-VEGF Treatment Response. Ophthalmology. 2016;123(11):2368-2375. 